\title{
Rechtsprechung
}

\section{Aktuelles aus der Rechtsprechung zu kunstrechtlichen Streitigkeiten}

\author{
Ines Zander*
}

Seit Ende des Jahres 2018/Anfang 2019 hat sich der Bundesgerichtshof zu wesentlichen kunstrechtlichen Themen gleich mehrfach geäußert. Im Fokus stand dabei der urheberrechtliche Schutz des Künstlers. Daneben beschäftigten auch beweisrechtliche Fragen den Bundesgerichtshof im Sommer 2019. Die richtungsweisenden Entscheidungen dürften erhebliche Auswirkungen für die Praxis haben und zeigen auch auf, wo gesetzgeberische Klarstellungen hilfreich wären.

\section{Fotos von gemeinfreien Kunstwerken scheitern am Urheberrecht bzw. dem musealen Besichtigungsvertrag}

- Das Spannungsfeld zwischen Bildern von gemeinfreien Kunstwerken und Urheberrecht ist bekannt und seit Jahren Gegenstand des Diskurses. Dabei geht es vor allem um solche Fotografien, die auf eine möglichst genaue Reproduktion des Motivs, also des meist gemeinfreien Kunstwerks, zielen und bei denen die urheberrechtliche Schutzfähigkeit aufgrund des technisch geprägten Herstellungsvorgangs fragwürdig erscheinen mag. Im Dezember 2018 äußerte sich der Bundesgerichtshof hierzu und schlug sich auf die Seite der Fotografen. In dem viel beachteten Fall "Museumsfotos"1 ${ }^{\text {1 }}$ beanspruchte die klagende Betreiberin des Reiss-Engelhorn-Museums in Mannheim Schutz gegen die Veröffentlichung von Fotos von gemeinfreien Kunstwerken. Sie stammten zum Teil aus einer Publikation des Hauses, die die beklagte Privatperson eingescannt hatte. Darüber hinaus hatte der Beklagte im Museum eigene Fotografien angefertigt, die er - ebenso wie die eingescannten Bilder - in das Internetportal Wikimedia hochlud. Das Reiss-EngelhornMuseum sah sich dadurch in seinen Rechten verletzt. Dieser Einschätzung gab der Bundesgerichtshof im Ergebnis Recht.

\section{a) Urheberrechtlicher Schutz erfasst auch Abbildungen von gemeinfreien Kunstwerken}

Für die eingescannten Fotos begründete er dies mit einer Urheberrechtsverletzung. Dafür sprach er den von den Mitarbeitern der Klägerin geschaffenen Bildern der Kunstwerke Lichtbildschutz nach $\S 72$ UrhG zu. ${ }^{2}$ Auch handwerkliche Leistungen ohne künstlerische Aussage könnten in den Schutzbereich des $§ 72$ UrhG fallen. ${ }^{3}$ Dies entspreche der gesetzgeberischen Vorstellung, nach der der Lichtbildschutz auch rein technische Leistungen, die keine besonderen Fähigkeiten des Lichtbild-

\footnotetext{
Dr. Ines Zander ist Rechtsanwältin in Hamburg.

BGH, Urt. 20.12.2018 - I ZR 104/17 = GRUR 2019, 284 mit Anm. Zech.

2 BGH, Urt. 20.12.2018 - I ZR 104/17 = GRUR 2019, 284, 286.

3 BGH, Urt. 20.12.2018 - I ZR 104/17 = GRUR 2019, 284, 286 f.
}

ners verlangten, erfasse, so der Bundesgerichtshof. ${ }^{4}$ In diesem Kontext setzte er sich auch mit der Gegenmeinung auseinander und führte aus, dass eine teleologische Reduktion des $\S 72$ UrhG nicht in Betracht komme. Entgegen einer Literaturmeinung schränkte der Bundesgerichtshof damit den Lichtbildschutz für Aufnahmen, die mit dem Ziel gefertigt wurden, eine möglichst große Ähnlichkeit mit dem Original zu erzielen, nicht ein. Denn der Lichtbildschutz hindere die Allgemeinheit nicht an der Auseinandersetzung mit einem gemeinfreien Werk, da er lediglich der Vervielfältigung entgegenstehe. ${ }^{5}$

\section{b) Fotografierverbot kraft musealen Besichtigungsvertrags}

In Bezug auf die vom Beklagten selbst angefertigten Fotos nahm der Bundesgerichtshof eine Verletzung des Besichtigungsvertrags an. Dabei folgte er der Klägerin in der Annahme, dass aus den Piktogrammen und der Benutzungsordnung ein wirksam in den Vertrag einbezogenes Fotografierverbot folge. Dem Einwand des Beklagten, die in dem Fotografierverbot liegende Benachteiligung sei unangemessen, folgte das Gericht hingegen nicht. Es bestehe ein berechtigtes Interesse der Betreiber von Museen für ein solches Verbot, das dem Schutz der Kunstwerke, dem ordnungsgemäßen Ablauf des Museumsbetriebs oder der Einhaltung rechtlicher Verpflichtungen des Museums dienen könne. ${ }^{6}$ Im Falle des Fotografierverbots stelle das Ordnungs- und Schutzinteresse eines Museumsbetreibers einen hinreichenden Gemeinwohlgrund dar.?

\section{c) Keine Stellungnahme zur Frage eines „Recht[s] am Bild der eigenen Sache"}

Nicht entscheidungserheblich war im Fall "Museumsfotos" hingegen die Frage nach einem „Recht am Bild der eigenen Sache", bei der es darum geht, ob der Eigentümer einer Sache auf Grundlage seiner eigentumsrechtlichen Befugnisse auch

BGH, Urt. 20.12.2018 - I ZR 104/17 = GRUR 2019, 284, 286 f.

$5 \quad$ BGH, Urt. 20.12.2018 - I ZR 104/17 = GRUR 2019, 284, 287.

BGH, Urt. 20.12.2018 - I ZR 104/17 = GRUR 2019, 284, 289 f.

BGH, Urt. 20.12.2018 - I ZR 104/17 = GRUR 2019, 284, 290. 
darüber bestimmen kann, wer die Sache fotografieren und die gefertigten Bilder vermarkten darf. Mit ebendieser Frage befasste sich im Sommer 2019 jedoch das Oberlandesgericht München, ${ }^{8}$ welches sich für eine Differenzierung aussprach, die daran anknüpfen soll, ob zum Fotografieren der beweglichen Sache widerrechtlich das Grundstück ihres Eigentümers betreten werden muss (dann liege eine Eigentumsverletzung vor) - oder eben nicht. ${ }^{9}$ Das Schaffen einer ausdifferenzierten Regelung obliege im Übrigen dem Gesetzgeber. ${ }^{10}$ Damit bemüht sich das Oberlandesgericht München, die Rechtsprechung des Bundesgerichtshofs zu Fotos unbeweglicher Sachen auf einen Sachverhalt mit beweglichen Werken zu übertragen. In der Konsequenz dürfte es demnach kein „Recht am Bild der eigenen Sache" geben. Maßgeblich wären vielmehr - wie die Entscheidung des Bundesgerichtshofs „Museumsfotos" zeigt die Zugangs- und Nutzungsabsprachen bzw. -bedingungen der das Kunstwerk bewahrenden Einrichtung.

\section{d) Konflikt zwischen Freihalteinteresse der Allgemeinheit und Interessen der Museen bleibt bestehen}

Die Entscheidung des Bundesgerichtshofs im Fall „Museumsfotos" bringt somit keine Abkehr von den bisherigen Grundsätzen. Es bleibt bei dem umstrittenen Lichtbildschutz für Reproduktionsfotografie, der in Schrifttum und Praxis kritisch gesehen wird. Ob hier der Gesetzeswortlaut über den Sinn und Zweck hinausgehende Konstellationen erfasst, bleibt Klarstellungsaufgabe des Gesetzgebers. Dabei gilt es einerseits, den Zugang der Allgemeinheit zu gemeinfreien Werken sicherzustellen und eine Monopolisierung zu vermeiden. Andererseits haben auch die Museumsbetreiber nicht nur ein Ordnungs- und Schutzinteresse. Vielmehr sorgen sie zugleich für die Unterhaltung der Museen und investieren in die Bewahrung der dort aufbewahrten Stücke. Damit ermöglichen sie es erst, dass sich die Besucher an den Kunstwerken erfreuen können. Einen möglichen Weg des Umgangs mit diesem Konflikt deutet die im Jahr 2017 ergangene Entscheidung der KFIR-Beschwerdekammer im Fall Gustav Vigeland ${ }^{11}$ an, die angesichts des besonderen kulturellen Werts der Skulpturen für Norwegen ausnahmsweise von einem Vorrang des Freihalteinteresses der Allgemeinheit ausging. Ein Regel-Ausnahme-Verhältnis, orientiert an der besonderen (nationalen) Bedeutung des Kunstwerks, könnte auch für die Fotos von gemeinfreien Kunstwerken zu überlegen sein.

\section{Richtungsweisung beim urheberrechtlichen Schutz des Künstlers gegen Zerstörung seiner Werke und Schmerzensgeld für Künstler}

Die Zerstörung eines Kunstwerks kann der Urheber nicht verhindern, sie unterfällt den Befugnissen des Eigentümers - so sah es bislang ein nicht unbeachtlicher Teil der Fachwelt. Dieser Ansicht hat der Bundesgerichtshof im vergangenen Februar 2019 widersprochen.

Damals hatte der Bundesgerichthof gleich über zwei Fälle ${ }^{12}$ zu entscheiden, in denen eine international bekannte Künstlerin sich gegen die Entfernung zweier von ihr geschaffener Installationen aus der Mannheimer Kunsthalle wendete. Streitgegenständlich waren zum einen das Werk "HHole (for Mannheim) ${ }^{u \mathbf{1 3}}$, eine multimediale und multidimensionale, mit allen Geschossdecken festverbundene Rauminstallation im Athene-Track der Kunsthalle Mannheim, zum anderen die Lichtinstallation „PHaradise ${ }^{\text {"14 }}$, die mit dem Dach des Billing-Baus der Kunsthalle Mannheim fest verbunden war. Hintergrund der zum Teil schon vollzogenen bzw. anstehenden Entfernung waren umfassende Umbauarbeiten der Kunsthalle. Nachdem sie in den Instanzen unterlegen war, zog die Klägerin mit ihrem Begehren vor den Bundesgerichthof - im Ergebnis blieb sie damit weitestgehend erfolglos. Dies begründete der Bundesgerichtshof damit, dass er die (drohende) Vernichtung der Installationen für rechtmäßig hielt, so dass keine Verletzung von § 97 UrhG vorlag.

\section{a) Schutzbereich des $\S 14$ UrhG erfasst auch die Zerstörung des Werks}

Bemerkenswert an der Entscheidung ist, dass der Bundesgerichtshof im Grundsatz von einer Eröffnung des Anwendungsbereichs des $\S 14$ UrhG ausgeht und sich damit der im Schrifttum vertretenen Ansicht anschließt, nach der die Vernichtung eines Werkoriginals als schärfste Form der Verletzung anzusehen sei. ${ }^{15}$ Zur Begründung betonte er, dass weder die Wortlautgrenze noch die gesetzgeberische Intention dieser Interpretation entgegenstünden. Vielmehr spreche der Zweck des § 14 UrhG dafür, den Urheber auch gegen die Vernichtung seiner Werke zu schützen. Denn das Urheberpersönlichkeitsrecht könne durch die Vernichtung eines Werks in besonderer Weise betroffen sein, da die Vernichtung das Fortwirken des Werks (als Ausdruck der Persönlichkeit seines Schöpfers) vereiteln oder erschweren könne. ${ }^{16}$ Überdies könne so auch grundrechtlichen

12 BGH, Urt. v. 21.2.2019 - I ZR 98/19 juris, abgedruckt u.a. GRUR 2019, 609; BGH, Urt. v. 21.2.2019 - I ZR 99/19 juris, abgedruckt u.a. ZUM 2019, 521.

13 BGH, Urt. v. 21.2.2019 - I ZR 98/19 juris.

14 BGH, Urt. v. 21.2.2019 - I ZR 99/19 juris.

15 BGH, Urt. v. 21.2.2019 - I ZR 99/17 juris Rn. 29 ff.; BGH, Urt. v. 21.2.2019 - I ZR 98/19 juris Rn. 30 ff.

16 BGH, Urt. v. 21.2.2019 - I ZR 99/17 juris Rn. 32; BGH, Urt. v. 21.2.2019 - I ZR 98/19 juris Rn. 33. 
Wertungen Rechnung getragen werden, da § 14 UrhG im Tatbestandsmerkmal der „berechtigten, geistigen oder persönlichen Interessen“ eine Interessensabwägung ermögliche. ${ }^{17}$

\section{b) Interessenabwägung nach $\S 14$ UrhG bei Baukunst in der Regel zugunsten des Eigentümers}

Die Ausweitung des Schutzbereichs half der Künstlerin in beiden Fällen jedoch im Ergebnis nicht. Der Bundesgerichtshof ging - wie das Berufungsgericht - zulasten der Klägerin davon aus, dass die Interessen des Urhebers am Fortbestand des Kunstwerkes bei Werken der Baukunst, bei mit Bauwerken unlösbar verbundenen Werken oder sonst grundstücksbezogenen Werken in aller Regel hinter den Interessen des Eigentümers an der anderweitigen Nutzung oder Bebauung und der damit verbundenen Zerstörung des Werks zurückstehen. Dies gelte auch vor dem Hintergrund, dass - anders als bei zerstörungsfrei entfernbaren Kunstwerken - die Rechte des Eigentümers völlig aufgehoben wären, wenn der Urheber einer mit einem Gebäude unlösbar verbundenen Installation deren Entfernung dauerhaft untersagen könne. ${ }^{18}$

\section{c) Aspekte für die Interessensabwägung}

Die Urteile des Bundesgerichtshofs zu der Rechtmäßigkeit der Entfernung von Kunstinstallationen aus der Kunsthalle Mannheim erzeugen Klarheit hinsichtlich des Schutzumfangs von $\S$ 14 UrhG. Sie werfen jedoch zugleich neue Frage auf. So dürften die Interessen der Beteiligten insbesondere dann anders zu bewerten sein, wenn das in Rede stehende Kunstwerk nicht unlösbar mit dem Gebäude verbunden ist. Diesbezüglich lassen sich den Entscheidungen jedoch für die Abwägung zu berücksichtigende Aspekte entnehmen wie die Frage, ob ein Unikat oder ein Werk aus einer Auflagenproduktion vorliegt, die Gestaltungshöhe und den Umstand, ob es sich um einen Gegenstand der zweckfreien oder der angewandten Kunst handelt. ${ }^{19}$ Auch kann relevant werden, ob der Künstler Gelegenheit hatte, das Werk zurückzunehmen oder Vervielfältigungsstücke davon anzufertigen. $^{20}$

\section{d) Zerstörung kann Schmerzensgeldanspruch auslösen}

Die gerade dargestellte Rechtsprechung bestätigte der Bundesgerichtshof in dem taggleich verkündeten Urteil im Fall „Minigolfanlage “21. Geklagt hatten hier zwei Künstler, von

17 BGH, Urt. v. 21.2.2019 - I ZR 99/17 juris Rn. 35; BGH, Urt. v. 21.2.2019 - I ZR 98/19 juris Rn. 36.

18 BGH, Urt. v. 21.2.2019 - I ZR 99/17 juris Rn. 35; BGH, Urt. v. 21.2.2019 - I ZR 98/19 juris Rn. 43.

19 BGH, Urt. v. 21.2.2019 - I ZR 99/17 juris Rn. 39; BGH, Urt. v. 21.2.2019 - I ZR 98/19 juris Rn. 39.

20 BGH, Urt. v. 21.2.2019 - I ZR 99/17 juris Rn. 41; BGH, Urt. v. 21.2.2019 - I ZR 98/19 juris Rn 41.

21 BGH, Urt. v. 21.2.2019 - I ZR I ZR 15/18, GRUR 2019, 619, nachfolgend: KG Berlin, Urt. v. 16.12.2019 - 24 U 173/15, aufrufbar unter BeckRS 2019, 34211. denen einer im Eingangsbereich einer Minigolfanlage eine Brunneninstallation mit dem Titel "Quelle des Sonnensystems" geschaffen hatte, während der andere im sogenannten „SternRaum" eine Sterninstallation kreiert hatte. Beide Installationen waren im Rahmen von Umbauarbeiten zerstört worden. Mit der Klage begehrten die Künstler Schmerzensgeld, was Landgericht und Berufungsgericht abgelehnt hatten. Der Bundesgerichtshof hob die Entscheidung der Vorinstanz auf und stellte auch hier klar, dass die Zerstörung der Installationen vom Schutz des § 14 UrhG erfasst ist. Für den Fall, dass die Vernichtung im Streitfall geeignet gewesen sei, die berechtigten geistigen und persönlichen Interessen der Künstler zu gefährden, müsse das Berufungsgericht weiter prüfen, ob die Voraussetzungen eines Schmerzensgeldanspruchs erfüllt seien. ${ }^{22}$ Das Kammergericht Berlin, an das die Sache zurückverwiesen wurde, sprach den Künstlern im weiteren Verlauf des Rechtsstreits Schmerzensgeld in Höhe von EUR 10.000,00 bzw. EUR 14.000,00 zu. ${ }^{23}$ Für die Interessensabwägung stellte der Senat darauf ab, dass es sich bei den Installationen um Unikate handele, die sich in ihrer konkreten Gestaltung deutlich von anderen Werken der Künstler unterschieden. ${ }^{24}$ Die Brunneninstallation weise eine Vielzahl von Einzelelementen auf, während die Sterninstallation durch die große Zahl der verknüpften, farbigen und fluoreszierenden Wollfäden und die bei der Rotation entstehenden Lichteffekte beeindrucke. ${ }^{25}$ Zudem seien beide Installationen - vergleichbar mit Bühnenbildern - der angewandten Kunst zuzuordnen, da sie einem Gebrauchszweck dienten (nämlich als Dekoration der Minigolfanlage). ${ }^{26}$ Auf Seiten der Beklagten seien bautechnische Gründe sowie das Interesse an einer Nutzungsänderung zu berücksichtigen. ${ }^{27} \mathrm{Zu}$ beachten sei weiter, dass die Installationen mit den Räumlichkeiten nicht unlösbar verbunden gewesen seien, sondern unter teilweiser schonender Zerlegung hätte abgebaut werden können ${ }^{28}$ und dass die Beklagten den Künstlern nicht Gelegenheit gegeben hatten, die Installationen selbst abzubauen und zurückzunehmen. ${ }^{29}$ Im Ergebnis rechtfertigten die von den Beklagten angeführten Interessen keinesfalls die Zerstörung der Installationen. Die Beklagten seien allenfalls berechtigt, die Werke unter "vorsichtiger Teilzerlegung“ zu entfernen und an einem sicheren Ort bis zur Abholung durch

22 BGH, Urt. v. 21.2.2019 - I ZR I ZR 15/18, GRUR 2019, 619, 619.

23 KG Berlin, Urt. v. 16.12.2019 - 24 U 173/15, aufrufbar unter BeckRS 2019, 34211.

24 KG Berlin, Urt. v. 16.12.2019 - 24 U 173/15, aufrufbar unter BeckRS 2019, 34211 Rn. 20.

25 KG Berlin, Urt. v. 16.12.2019 - 24 U 173/15, aufrufbar unter BeckRS 2019, 34211 Rn. $22 \mathrm{f}$.

26 KG Berlin, Urt. v. 16.12.2019 - 24 U 173/15, aufrufbar unter BeckRS 2019, 34211 Rn. 24.

27 KG Berlin, Urt. v. 16.12.2019 - 24 U 173/15, aufrufbar unter BeckRS 2019, 34211 Rn. $27 \mathrm{ff.}$

28 KG Berlin, Urt. v. 16.12.2019 - 24 U 173/15, aufrufbar unter BeckRS 2019, 34211 Rn. 31.

29 KG Berlin, Urt. v. 16.12.2019 - 24 U 173/15, aufrufbar unter BeckRS 2019, 34211 Rn. 32 
die Kläger oder deren Ablehnung zu verwahren. Dies allerdings erst, nachdem sie den Klägern Gelegenheit gegeben haben, die Werke selbst abzubauen und zu entfernen. ${ }^{30}$

\section{e) Auswirkungen für die Praxis}

Auswirkungen hat diese Rechtsprechung auf den Umgang mit unlösbar, lösbar und nicht mit dem Gebäude verbundenen Kunstwerken. Die Interessensabwägungen kann in jeder dieser Kategorien unterschiedlich ausfallen - in der Regel im ersten Fall zugunsten des Grundstückeigentümers, ansonsten zugunsten des Künstlers. Allerdings können Einzelumstände zu anderen Ergebnissen führen. Beispielweise könnte der künstlerische Rang des in Rede stehenden Kunstwerks Bedeutung erlangen. Vor diesem Hintergrund erscheint es ratsam, die Frage des Umgangs mit nicht mehr benötigten Kunstwerken vertraglich zu regeln.

\section{Beweislastverteilung und sekundäre Darlegungs- last bei der Ersitzung gestohlener Bilder}

Die Erfolgsaussichten von Rechtssachen, die Kunstwerke betreffen, hängen oft eng mit Beweisfragen und Sorgfaltspflichten zusammen. Einen „Lehrbuchfall“ zum Zusammenspiel von materiellem Recht mit Fragen der prozessualen Durchsetzbarkeit hatte der Bundesgerichtshof im Sommer 2019 zu entscheiden.

Streitgegenständlich waren in diesem Verfahren ${ }^{31}$ zwei Ge- $^{-}$ mälde mit den Titeln „Frau im Sessel“ und „Blumenstrauß", die von dem Maler Hans Purrmann stammen sollten. Der Kläger, Enkel des Malers, behauptete, dass die Bilder seiner Mutter geschenkt und bei einem Einbruch in das Anwesen seiner Eltern im Jahr 1986 entwendet worden seien; der Beklagte berief sich demgegenüber auf Ersitzung. Das Berufungsgericht erachtete die Klage für unbegründet und bestätigte die Entscheidung des Landgerichts. Dies hielt der rechtlichen Nachprüfung durch den Bundesgerichtshof nicht stand.

\section{a) Nachweis der Echtheit der Bilder und der Eigentümerstellung der Mutter des Klägers}

Der Bundesgerichtshof ging zunächst davon aus, dass das Berufungsgericht Beweisangebote des Klägers zur Echtheit der Gemälde übergangen habe. Er sah in dem Vortrag des Klägers, dass der Leiter des Purrmann-Archivs in München, der für den Maler Purrmann zwei Werkverzeichnisse erstellt habe, die Echtheit der Gemälde bei einer polizeilichen Vernehmung bestätigt habe, und der zum Nachweis angebotenen Vernehmung dieses Leiters, einen tauglichen Beweisantritt. ${ }^{32}$

30 KG Berlin, Urt. v. 16.12.2019 - 24 U 173/15, aufrufbar unter BeckRS 2019, 34211 Rn. 39.

31 BGH, Urt. v. 19.07.2019 - V R 255/17 juris, abgedruckt u.a. ZUM 209, 845 .

32 BGH, Urt. v. 19.07.2019 - V R 255/17 juris Rn. 13 ff.
Auch habe das Berufungsgericht verkannt, dass aufgrund des für das Revisionsverfahren zu unterstellenden Besitzes nach § 1006 Abs. 2 BGB zu vermuten sei, dass die Mutter des Klägers Eigentümerin der streitgegenständlichen Bilder gewesen sei. ${ }^{33}$

\section{b) Beweislast für den bösen Glauben im Rahmen von § 937}

\section{Abs. 2 BGB bei gestohlenen Sachen}

Im Weiteren befasste sich der Bundesgerichtshof mit der Beweislastverteilung im Rahmen von $\S 937$ Abs. 2 BGB bei gestohlenen Sachen. Im Grundsatz trage der Kläger die Beweislast für die Bösgläubigkeit des Beklagten, was auch im Fall einer gestohlenen oder sonst abhandengekommenen Sache gelte. ${ }^{34}$ Damit erteilte der Bundesgerichtshof der gegenteiligen Auffassung in der obergerichtlichen Rechtsprechung eine Absage, nach der den Ersitzenden die volle Beweislast treffen soll, wenn die Sache einem früheren Besitzer gestohlen wurde oder sonst abhandengekommen ist. Zur Begründung führte der Bundesgerichtshof aus, dass nach seiner bisherigen Rechtsprechung zwar dem früheren Besitzer, dem die Sache abhandengekommen ist, ausnahmsweise auch gegenüber dem derzeitigen Besitzer die Eigentumsvermutung nach $\S 1006$ Abs. 2 BGB zukommt, so dass letzterer die Voraussetzungen eines Erwerbstatbestands darlegen und beweisen muss, wovon er ohne das Abhandenkommen nach § 1006 Abs. 1 Satz 1 BGB enthoben wäre. ${ }^{35}$ Für eine darüber hinausgehende Beweislastumkehr, die angesichts des klaren Wortlauts des § 937 Abs. 2 BGB nur über eine teleologische Reduktion möglich wäre, sei indes kein Raum. ${ }^{36}$ Der Gesetzgeber habe sich bewusst für eine Regelung entschieden, bei der der gute Glaube des Ersitzenden nicht zur Voraussetzung der Norm gemacht werde. Stattdessen kann das Fehlen des guten Glaubens ausnahmsweise die Ersitzung ausschließen. Das mit dieser Regelung verfolgte Ziel des Gesetzgebers sei folglich der Schutz des guten Glaubens des Erwerbers auch und gerade in Bezug auf abhandengekommene Sachen, unabhängig von der Art des Erwerbs. ${ }^{37}$ Diesem Ziel entspreche die Beweislastverteilung in $\S 937$ Abs. 2 BGB. ${ }^{38}$

\section{c) Kein kunstmarktspezifischer Sorgfaltsmaßstab für Laien beim Kauf von Kunstwerken}

Anschließend streifte der Bundesgerichtshof auch die Frage, ob im Kunsthandel für die Beteiligten ein spezifischer Sorgfaltsmaßstab anzulegen sei, weil die Veräußerungsvorgänge wertvoller Kunstgegenstände öffentliche Aufmerksamkeit weckten und allgemein bekannt sei, dass die Eigentumslage bei Kunstwerken oftmals zweifelhaft sei und in Fachkreisen kritisch diskutiert werde. ${ }^{39}$ Zu einer Entscheidung dieser Streitfrage kam es

33 BGH, Urt. v. 19.07.2019 - V R 255/17 juris Rn. 22 ff.

34 BGH, Urt. v. 19.07.2019 - V R 255/17 juris Rn. 38 ff.

35 BGH, Urt. v. 19.07.2019 - V R 255/17 juris Rn. 41.

36 BGH, Urt. v. 19.07.2019 - V R 255/17 juris Rn. 42.

37 BGH, Urt. v. 19.07.2019 - V R 255/17 juris Rn. 42.

38 BGH, Urt. v. 19.07.2019 - V R 255/17 juris Rn. 42.

39 BGH, Urt. v. 19.07.2019 - V R 255/17 juris Rn 46. 
jedoch nicht, da - so der Bundesgerichtshof - im Rahmen von $\S 937$ Abs. 2 BGB eine Pflicht zur Nachforschung jedenfalls für Laien nicht Voraussetzung sei. ${ }^{40}$ Der Erwerber könne allerdings bösgläubig sein, wenn besondere Umstände seinen Verdacht erregen müssten und er Nachforschungen unterlässt. ${ }^{41}$

\section{d) Sekundäre Darlegungslast hinsichtlich der Bösgläubigkeit}

Der Bundesgerichtshof hat sich dann noch zu der Möglichkeit geäußert, die Bösgläubigkeit des Beklagten als zugestanden zu werten. Den Ersitzenden treffe eine sekundäre Darlegungslast, wenn die primär darlegungsbelastete Partei keine nähere Kenntnis der maßgeblichen Umstände und zudem keine Möglichkeit zur weiteren Sachverhaltsaufklärung habe, während dem Prozessgegner nähere Angaben ohne weiteres möglich und zumutbar seien. ${ }^{42}$ Der vom früheren Besitzer zu führende volle Beweis des bösen Glaubens des Besitzers bei Besitzerwerb sei als geführt anzusehen, wenn er die vom Besitzer behaupteten Erwerbsumstände widerlege; es sei nicht erforderlich, dass alle denkbaren anderen Erwerbsvorgänge bzw. -tatbestände widerlegt würden. ${ }^{43}$ Vor diesem Hintergrund sei nicht auszuschließen, dass das Berufungsgericht zu einem anderen Ergebnis gekommen wäre, wenn es den richtigen Maßstab angelegt hätte. Zweifel folgten bereits daraus, dass der Beklagte wechselnde Angaben zum Besitzerwerb gemacht habe. ${ }^{44}$ Jedenfalls fehle es an einer richterlichen Würdigung, ob der konkrete Vortrag des Beklagten als widerlegt anzusehen sei oder nicht. ${ }^{45}$ Der Kläger habe Hinweise vorgetragen, die dafür sprechen könnten, dass dem Beklagten der Wert der Gemälde bekannt war, wie den Versuch, die Gemälde in der Schweiz und nicht in Deutschland zu verkaufen, die Weigerung, die Bilder durch das Purrmann-Archiv in München begutachten zu lassen, und die Aussage der Tochter des Beklagten, dass die Herkunft der Bilder in der Familie nie zum Gesprächsthema gemacht worden sei, es aber Gedanken dazu gegeben habe, ob jemand einbrechen könne, um die Bilder zu stehlen.

\section{e) Bedeutung für die Praxis}

Das Urteil des Bundesgerichtshofs beleuchtet die Bedeutung der Beweisführung in Kunstrechtsstreitigkeiten und zeigt die Wichtigkeit einer sorgfältigen Prozessführung. Begrüßenswert ist die Klarstellung, dass es auch bei gestohlenen oder sonst abhandengekommenen Kunstwerken bei der geltenden Beweisverteilung bleibt. Wie das Urteil illustriert, können im Einzelfall sekundäre Darlegungslasten dem früheren Besitzer zur Hilfe kommen. Der Fortgang des Falls bleibt abzuwarten.

\section{Fazit}

Die dargestellten Entwicklungen deuten eine künstlerfreundliche Rechtsprechungstendenz an, es bleiben jedoch auch fortbildungsbedürftige Themen, die ggf. von dem Gesetzgeber aufgriffen werden könnten. Die urheberrechtliche Vertragsgestaltung dürfte in der Praxis helfen, Konflikte zu vermeiden. 


\title{
Literatur
}

\section{Moritz Holfelder: Unser Raubgut}

\author{
Eine Streitschrift zur kolonialen Debatte
}

\section{Robert Kirchmaier*}

- Moritz Holfelder greift ein in den letzten Jahren verstärkt ins öffentliche Bewusstsein gerücktes Thema auf, das er mit seiner - so der Untertitel - „Streitschrift zur kolonialen Debatte" raschen Lösungen zuführen möchte. Nicht dass er für sich reklamieren würde, für die in diesem Zusammenhang zu klärenden Fragen bereits die passenden Antworten zu haben. Er möchte aber wachrütteln und Anstöße dazu geben, die Fragen nach dem Umgang mit Kulturgütern aus kolonialen Kontexten in europäischem Besitz anzugehen und im Interesse der Herkunftsländer zu klären. Der Titel „Unser Raubgut“ ist provokativ gewählt, darüber hinaus aber durchaus geeignet, zunächst für Unsicherheit und Verwirrung zu sorgen. Der Begriff „Raubgut“ ist in den Restitutionsdebatten der letzten 25 Jahre verbunden mit der Rückgabe von Kulturgütern, die während der nationalsozialistischen Herrschaft vornehmlich jüdischen Eigentümern abgepresst und entzogen wurden. Die Übertragung der Begrifflichkeit auf koloniale Kontexte erscheint wenig glücklich.

Holfelder stellt an den Beginn seiner Ausführungen den im Jahr 1953 erschienenen Dokumentarfilm „Les Statues meurent aussi“ der Regisseure Alain Resnais und Chris Marker, der sich kritisch mit der Herkunft afrikanischer Kunst und den kolonialen Zusammenhängen befasste und prompt zunächst der Zensur unterfiel - ein Thema, über das man lange Zeit und nicht nur in Frankreich nicht sprach, bis der französische Staatspräsident Emmanuel Macron im November 2017 in der Universität von Ouagadougou einen Paradigmenwechsel ankündigte, der ganz Europa aufhorchen ließ. Holfelder analysiert diesen nahezu revolutionären Ansatz des französischen Präsidenten und verfolgt die Aufnahme der Neuausrichtung sowohl auf politischer Ebene wie auch in den einschlägigen Kulturinstitutionen. Dabei gelangt er zu der Einschätzung, dass der leidenschaftliche Appell Macrons zunächst zwar gehört und positiv aufgenommen wurde, im weiteren Verlauf aber nicht mit der gebotenen Durchsetzungskraft weiterverfolgt wurde. Hol-

\footnotetext{
* Robert Kirchmaier ist Oberlandesanwalt bei der Landesanwaltschaft Bayerns und Mitglied im Wissenschaftlichen Beirat der KUR.
}

felder vermisst hier die Entschlossenheit und den nötigen Biss, eine Auffassung, der man nicht unkritisch folgen muss, wenn man ins Kalkül zieht, dass hier feste Strukturen und Überzeugungen bzw. Überlieferungen in Zweifel zu ziehen und auf den Prüfstand zu stellen sind, die sich mit einem gewissen Beharrungsvermögen erstmals Veränderungen und Forderungen nach solchen Veränderungen zu entziehen versuchen - ein relativ normaler Vorgang, der schlicht gesagt seine Zeit braucht. Holfelder geht der Frage nach, woran es liegen mag, dass es nicht zu einem rascheren Umdenken und Handeln im Interesse der Herkunftsländer kommt. Dabei wird deutlich, dass er sich mit den Fragen intensiv und kenntnisreich beschäftigt hat und unterschiedliche Ansätze berücksichtigt. An Beispielen lässt er es nicht mangeln, erwähnt werden sollen an dieser Stelle insbesondere die legendären Bronzen aus Benin.

Holfelder berichtet auch von einer Reihe gelungener Rückgaben von Kulturgütern aus kolonialen Kontexten. Besonders bemerkenswert dabei ist, dass er hier immer wieder authentische Stimmen von Vertretern der Ursprungsländer ausführlich in der Diskussion zu Wort kommen lässt, was letztlich für ein gegenseitiges Verstehen von Positionen und ein besseres Verständnis unerlässlich ist.

Relativ ausführlich bezieht Holfelder die aktuelle Entwicklung in Deutschland mit ein, die sich vor allem am Berliner Humboldt Forum festmachen lässt. Hier spart er nicht mit Kritik, liefert darüber hinaus aber auch eine ganze Reihe hochinteressanter Hintergrundinformationen.

Seine Streitschrift endet mit sieben Vorschlägen zum Umgang mit der kolonialen Vergangenheit, die in ihrer Allgemeinheit sicherlich weithin Zustimmung finden werden. Etwa die Forderung nach intensiverer, durch persönliche Begegnungen geprägte Auseinandersetzung mit Afrika, einen umfassenden Austausch mit den Herkunftsgesellschaften auf Augenhöhe ohne europäische Beanspruchung der Deutungshoheit und verbunden mit der Bereitschaft einander zuzuhören.
Christoph Links Verlag,

Berlin 2019

224 Seiten,

43 s/w Abbildungen,

Broschur, 18,00€ 
Auch die grundlegende Forderung, sich mit der eigenen kolonialen Vergangenheit kritisch auseinanderzusetzen, wird wenig Widerspruch finden. Vor dem Hintergrund der 1998 verabschiedeten „Washington Principles" stellt Holfeld zu Recht die Forderung nach einer entsprechenden Erklärung für Kulturgüter aus kolonialen Texten in den Raum. Die weitreichendste, allerdings einer Streitschrift auch entsprechende Forderung geht dahin, sich zu einem grundlegenden Bekenntnis zur Restitution der durch Unrechtshandlungen oder asymmetrische Herrschaftsverhältnisse erlangten Kulturgüter durchzuringen, einer symbolischen Geste mit Überzeugungskraft.

Wer sich mit der Frage der Rückgabe von Kulturgütern aus kolonialen Kontexten auseinandersetzen möchte tut gut daran, das Buch von Moritz Holfelder zu Rate zu ziehen. Es ist eine ideale Einstiegsliteratur, eingängig geschrieben mit anschaulichen Beispielen. Holfelder bringt die Problematik auf den Punkt und spitzt auch dort zu, wo es wehtun mag. Das Buch ist ein leidenschaftlicher Appell, sich der historischen Verantwortung zu stellen und nach Lösungen im gemeinsamen Austausch mit den Herkunftsländern zu suchen. 